\title{
Tumeurs Cerebrales de l'enfant en milieu Canadien-Francais
}

\author{
F. RENEVEY, Y. AMYOT, G. GEOFFROY, M. VANASSE
}

SUMMARY: Between 1964 and 1976, we have seen 197 cases of cerebral tumors in children. We have studied the frequency of each histological group of tumors, their localization, the results of the clinical investigation, their clinical manifestations and the prognosis for each group.

This study has confirmed that in children brain tumors are more frequently. localized in the posterior fossa (56.4\%). As in other series, tumors derived from the glia represent nearly $80 \%$ of the total. Clinically, increased intra-cranial pressure is the most frequent manifestation of posterior fossa lesion (75\% of c'ases) while cerebellar involvement was seen in $60 \%$ of these childdren. Brain stem lesions and supratentorial tumors are more insidious

RÉSUMÉ: Au cours d'une période 12 ans, nous avons eu l'occasion de traiter 197 cas de tumeurs c'érébrales chez l'enfant. Nous avons étudié la fréquence des différents types histologiques, la localisation de ces tumeurs, leurs manifestations cliniques, l'apport diagnostique des divers moyens d'investigation et, enfin, le pronostic de chaque groupe de tumeur.

Cette étude nous a permis de confirmer la localisation préférentielle des tumeurs cérébrales de l'enfant au niveau de la fosse postérieure $(56.4 \%)$ ainsi que la fréquence très élevée des lésions tumorales issues de la glie, soit près de $80 \%$. Sur le plan clinique, les tumeurs de la fosse postérieure se manifestent surtout par un tableau dhypertension intra-crânienne $175 \%$ des cas) ainsi que par un syndrôme cérébelleux in their presentation and their clinical manifestations more diversified.

The EEG appeared useful in the investigation of brain tumors and showed irregularities in $78 \%$ of our patients. However, a more specific diagnosis required more invasive techniques such as angiograph.', pneumoencephalography or ventriculograph.'

As in previous series, the mortality rate remained high in the children w'e have followed, particularly for some groups of tumors (glioblastoma, medulloblastoma, brain stem lesions). It is to be hoped that with the introduction of the CT Scan, the improvement of neurosurgical techniques and the use of immuno and chemotherap., the prognosis may be improved.

dans $60 \%$ des cas. Le mode de présentation clinique des tumeurs du tronc et supratentorielles est généralement plus insidieux et leurs manifestations plus diversifiées.

Quoiqu'un diagnostic plus précis repose sur l'utilisation de techniques plus invasives, il faut souligner la valeur de l'électroencéphalographie qui s'est avérée positive dans $78 \%$ des cas.

Tout comme dans les séries similaires, le taux de mortalité, dans lensemble, demeure élevé chez les enfants que nous avons suivis. Il est d̀ espérer qu'un diagnostic plus précoce, grâce à la tomodensitométrie, ainsi que l'amélioration des techniques neuro-chirurgicales et, possiblement, l'introduction de limmuno et de la chimiothérapie puissent améliorer le pronostic.
From the Service de Neurologie, Département de Pédiatrie, Hôpital Sainte-Justine, Université de Montréal. Montréal. Québec, Canada.

Reprint requests to tirés à part: Dr. Guy Geoffroy, Hôpital Ste-Justine, 3175 Chemin Côte Ste-Catherine. Montréal, Québec, Canada, H3T 1 C5.

\section{INTRODUCTION}

Par leur fréquence, les tumeurs cérébrales viennent au second rang des néoplasies après les leucémies, et au premier rang des tumeurs solides dans la population pédiatrique américaine (Young et Coll., 1975). C'est donc un problème important comme en témoigne l'abondante littérature consacrée à ce sujet. Le but de la présente étude rétrospective s'étendant sur une période de 13 ans, est de comparer nos données aux séries les plus représentatives de cette littérature. Nous présenterons donc des statistiques sur:

- la fréquence et la répartition des différents types histologiques de tumeurs cérébrales.

- la sémiologie clinique.

- les investigations para-cliniques.

-l'incidence selon l'âge et le sexe.

- le pronostic des tumeurs les plus fréquentes.

\section{SELECTION DU MATERIEL}

Cette étude inclut tous les patients de 0 à 20 ans, admis à l'hôpital SainteJustine de Montréal entre le ler janvier 1964 et le 31 décembre 1976 avec un diagnostic de tumeur intracrânienne. Nous avons exclu les anomalies kystiques congénitales, les malformations vasculaires, les abcès et les hématômes.

\section{RESULTAT}

Données générales: Nos critères de sélection nous ont permis de constituer une série de 197 cas de tumeurs cérébrales comprenant 102 garçons (51.8\%) et 95 filles (48.2\%). 138 de ces enfants, c'est-à-dire $70 \%$ étaient âgés de 9 ans ou moins. 85 tumeurs ont une localisation supra-tentorielle $(43.6 \%)$ et 112 une localisation infra-tentorielle $(56.4 \%) .125(63.5 \%)$ sont situées sur la ligne médiane (supra ou infratentorielles). 
A la fin de cette étude, 89 enfants étaient décédés, ce qui représente un taux de mortalité de 45\%. Nous avions une relance suffisante pour 78 enfants dont nous détaillerons plus loin la durée et la qualité de survie. Par ailleurs, nous manquons d'informations concernant l'évolution de 30 enfants $(15.2 \%)$.

\section{HISTOLOGIE}

Nous utiliserons pour ce travail la classification proposée par Rubinstein en 1972. Le tableau numéro 1 donne une répartition globale des tumeurs cérébrales dans notre série selon les différents types histologiques. Sur les 197 cas, $33(16.7 \%)$ n'ont pas eu de diagnostic histologique, dont 18 tumeurs du tronc cérébral (documentées par les examens neuro-radiologiques) et 15 lésions inextirpables ou dont la localisation ne permettait pas une biopsie et 1 cas dont la vérification post-mortem n'a pu être obtenue.

On peut constater que les tumeurs issues de la neuroglie sont les plus fréquentes $(54.5 \%)$ suivies par les tumeurs d'origine neuronale ou de cellules bipotentielles surtout constituées par le médulloblastome $(5.5 \%$ à lui seul). Ces deux groupes constituent donc ensemble $75.3 \%$ des cas rapportés dans cette série. Chiffres comparables à ceux rapportés dans d'autres séries comme en témoigne le tableau 2.
TABLEAU 1

Repartition histologique des 164 T. C.

I Tumeurs des cellules neurogliales

Astrocytome (I et II)

Astroblastome (III et IV)

Ependymome

Oligodendrogliome

II Tumeurs d'origine neuronale et primairement bipotentielle Medulloblastome

Neuroblastome

III Tumeurs d'origine mésodermique

Origine méningée: méningiome

$$
\begin{aligned}
& \text { kyste leptoméningé } \\
& \text { sarcome méningé }
\end{aligned}
$$

Angiosarcome

IV Tumeurs des nerfs craniens

Gliome du nerf optique

V Tumeurs des plexus choroides Papillome

VI Tumeurs de la région pinéale Germinome

Teratome malin

VII Tumeurs secondaires à une anomalie du développement

Tératome embryonnaire malin

Rétinoblastome

Kyste dermoide

Craniopharyngiome

VIII Tumeurs secondaires

Séminome

Lymphosarcome

Hodgkin

TABLEAU 2

\begin{tabular}{|c|c|c|c|c|}
\hline & $\underset{\left(N^{*}: 671\right)}{\operatorname{MATSON}}(2)$ & $\begin{array}{l}\text { GJERRIS (23) } \\
\text { (N:281) }\end{array}$ & $\underset{(N: 124)}{\text { ONOYAMA }}$ & $\underset{\left(N^{*}: 164\right)}{\text { NOTRE ETUDE }}$ \\
\hline \multirow[t]{2}{*}{$\begin{array}{l}\text { ASTROCYTOME } \\
\text { (I et II) }\end{array}$} & $30.1 \%$ & $39.8 \%$ & $30.6 \%$ & $33.5 \%$ \\
\hline & & $\begin{array}{l}\text { I: } 13.9 \% \\
\text { II: } 25.9 \%\end{array}$ & $\begin{array}{l}\text { I: } 10.5 \% \\
\text { II: } 20.1 \%\end{array}$ & $\begin{array}{l}\text { I: } 11.5 \% \\
\text { II: } 22.0 \%\end{array}$ \\
\hline $\begin{array}{l}\text { ASTROBLASTOME } \\
\text { (III et IV) }\end{array}$ & $9.5 \%$ & $2.8 \%$ & $12.1 \%$ & $16.5 \%$ \\
\hline MEDULLOBLASTOME & $20.7 \%$ & $17.8 \%$ & $16.1 \%$ & $16.5 \%$ \\
\hline EPENDYMOME & $9.8 \%$ & $12.4 \%$ & $10.5 \%$ & $3.7 \%$ \\
\hline CRANIOPHAR YNGIOME & $10.1 \%$ & $5.7 \%$ & $8.1 \%$ & $5.5 \%$ \\
\hline
\end{tabular}

Tableau Comparatif des T.C. les plus Frequentes

$\mathrm{N}$ : Total de la série

$\mathrm{N}^{*}$ : Nombre avec histologie connue 


\section{SIGNES ET SYMPTOMES CLINIQUES}

Le tableau numéro 3 montre la nature et la fréquence des symptômes et signes cliniques les plus fréquents rencontrés dans les tumeurs cérébrales. Il est bien connu que la séméiologie dépend avant tout de la localisation et de lextension de la lésion plus que de sa nature histologique (Matson, 1969). Pour cette raison, nous avons établi l'incidence des symptômes et signes présents au moment où le diagnostic était posée en fonction de la localisation de la tumeur.

Les tumeurs de la fosse postérieure, à l'exclusion de celles du tronc cérébral, se présentent essentiellement avec une symptomatologie d'hypertension intra-crânienne et un syndrôme cérébelleux alors que les autres signes et symptômes neurologiques sont beaucoup moins fréquents. Notons toutefois que la douleur de la nuque et les symptômes d'irritation méningée ont été retrouvés dans $16 \%$ des cas. Les tumeurs du tronc par contre se manifestent plus fréquemment par une atteinte des nerfs crâniens, un syndrôme pyramidal ainsi qu'un syndrôme cérébelleux. Les symptômes d'hypertension intracrânienne sont nettement plus rares que pour les autres localisations et l'oedème papillaire ne fùt retrouvé que dans $7.7 \%$ des cas.

Enfin, les tumeurs à localisation supra-tentorielle se révèlent également le plus volontiers par une hypertension intracrânienne, mais la symptomatologie d'accompagnement est variée. Le syndrôme cérébelleux est plus rare que pour les deux autres localisations $(9 \%)$. Il existe cependant des troubles du caractère et de la conscience dans $31 \%$ des cas, des convulsions dans $25 \%$ des cas, des atteintes de la vision chez $25 \%$ des malades par lésions de la lle paire, du chiasma ou des voies optiques. Une atteinte des nerfs crâniens, principalement des nerfs oculo-moteurs est également fréquente $(22 \%)$. Enfin, des troubles endocriniens ont été notés chez $11 \%$ des cas, manifestations qui sont secondaires aux crânio-pharyngiomes et aux tumeurs de la région thalamique.

\section{INVESTIGATION PARA-CLINIQUE}

Les radiographies standards $d u$ crâne ont été positives dans environ $60 \%$ des cas. 93 sur 197 présentaient des signes radiologiques d'hypertension intra-crânienne. 15 fois seulement on pouvait distinguer des calcifications dont 7 étaient situées dans la région sellaire ou supra-sellaire. 152 des 190 électroencéphalogrammes initiaux pratiqués ont montré des anomalies diverses soit un pourcentage de $78 \%$.

La pneumoencéphalographie gazeuse (fractionnée ou non), la ventriculographie gazeuse ou iodographie ont été pratiquées selon les indications propres à chaque cas.

Tableau NNo3

\section{CLINIQUE EN REGARD DE LA LOCALISATION}

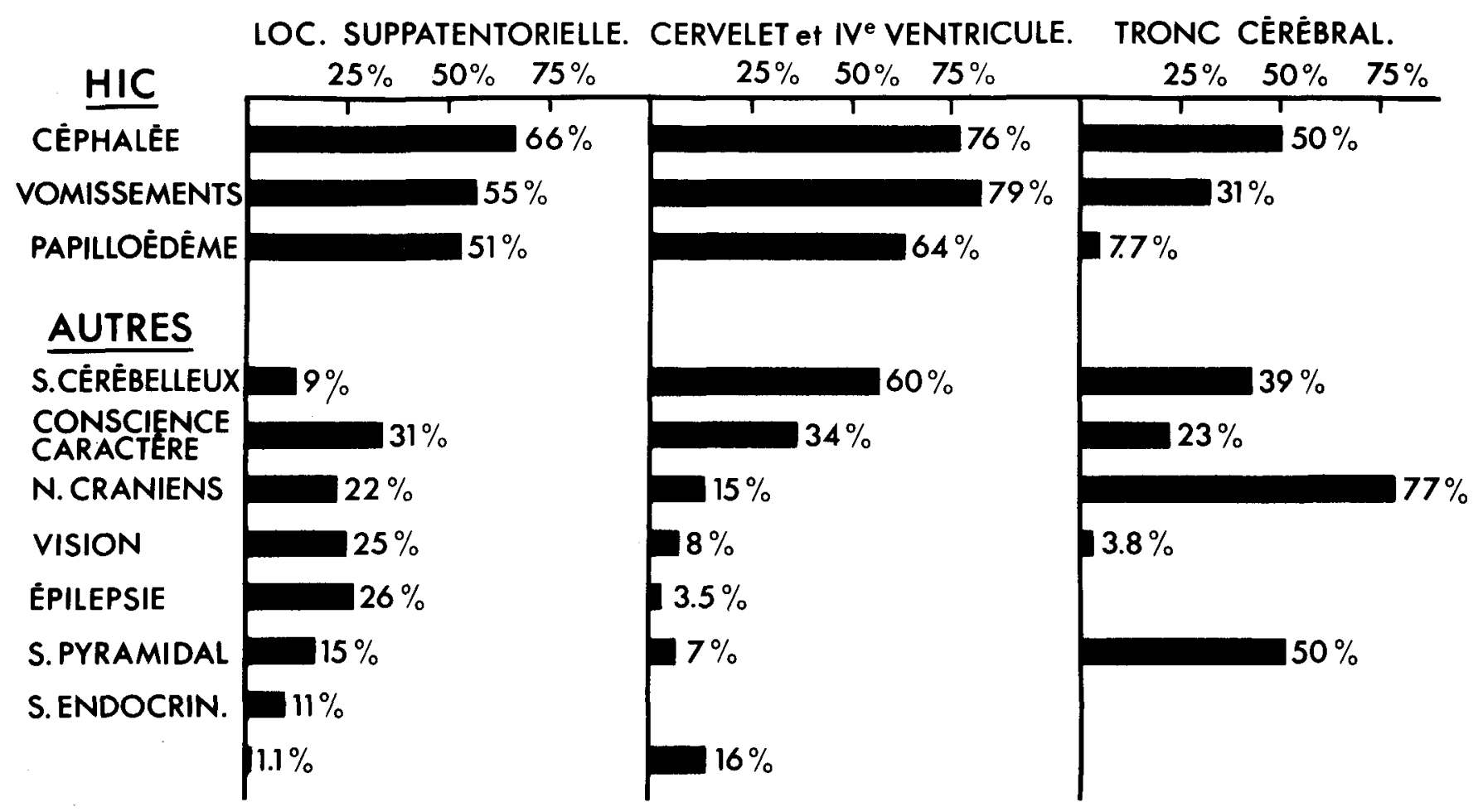


L'ensemble de ces examens s'est révélé pathologique dans 159 cas sur 197 (81\%). L'angiographie cérébrale n'a pas été pratiquée de routine mais chez. 115 malades, elle a été décrite comme anormale. L'échoencéphalographie et la gammaencéphalographie n'ont été utilisées que dans un nombre trop restreint de cas pour que nous puissions tirer des conclusions. De même, comme cette étude a été terminée en 1976, très peu d'enfants ont bénéficié de la tomodensitométrie cérébrale assistée par l'ordinateur (Emi-Scan) qui est devenue depuis lors un élément important dans le dépistage des tumeurs cérébrales.

\section{ANALYSE DES GROUPES HISTOLOGIQUES LES PLUS IMPORTANTS}

1. Astrocytomes (degrè de malignité l et II)

A) Astrocytomes supra-tentoriels:

Ce groupe est formé de 19 tumeurs (9.6\% du total) dont 8 étaient situées sur la ligne médiane (IIIe ventricule, région supra-sellaire, région thalamique, région pinéale) et 11 en périphérie (ventricules latéraux et hémisphères). L'âge moyen était de 8 ans et 2 mois (de 6 mois à 12 ans). Il y avait 12 filles et 7 garçons. Le délai moyen entre le début des symptômes et l'établissement du diagnostic était de 6,5 mois, indépendamment de la localisation de la tumeur.

La mortalité dépend évidemment beaucoup de la localisation qui peut limiter l'excision chirurgicale. Pour les tumeurs de la ligne médiane, 7 malades sur 8 sont décédés dont un en période post-opératoire. Par contre, pour les lésions hémisphériques, 3 malades sur 11 sont décédés, dont un en période post-opératoire. La moyenne de survie est de 4 ans et 7 mois et 4 des 9 survivants présentent des sequelles acceptables sous forme d'hémiparésie et/ou d'épilepsie focale, bien contrôlée par la médication. Deux enfants ont des signes de récidive de la lésion.

B) Astrocytomes infra-tentoriels: (à l'exclusion des tumeurs du tronc cérébral).

Il s'agit du groupe le plus important puisqu'il est constitué de 34 enfants ( $17.2 \%$ du total). L'âge moyen se situe à 7 ans et 6 mois ( 1 an et 5 mois à 13 ans et 5 mois). Il y a 20 garçons et 14 filles. Le délai moyen jusqu'à l'établissement du diagnostic a été de 3,8 mois. 13 de ces tumeurs étaient situées dans les hémisphères cérébelleux et 21 sur la ligne médiane (vermis et IVe ventricule).

Tout ont été opérés et 7 ont eu de plus une radiothérapie post-opératoire. Nous n'avons pas de relance chez 4 enfants. La mortalité globale dans le groupe des patients suivis a été de 4 sur $31(13 \%)$ dont 1 en période postopératoire $(3.2 \%)$. La survie moyenne actuelle est de 3 ans et 5 mois ( 1 an et 10 mois à 9 ans et 2 mois). Deux enfants présentent des signes de récidive alors que l'on note des sequelles neurologiques chez $16(51 \%)$ des survivants. Chez 14 de ces enfants, les sequelles sont mineures et chez 2 d'entre eux, elles sont importantes.

\section{Astroblastomes (degré de} malignité III et IV)

A) Astroblastomes supra-tentoriels:

Ce groupe de 13 enfants $(6.6 \%)$ comprend 7 garçons et 6 filles dont l'âge moyen est de 9 ans et 7 mois. Le délai entre le début des symptômes et le diagnostic a été de 2 mois et demi. Le pronostic est particulièrement sombre quels que soient la localisation ou le type de traitement. En dehors de 2 enfants dont nous ne connaissons pas l'évolution, tous sont décédés. Les enfants dont la tumeur était située sur la ligne médiane avaient un délai moyen entre le diagnostic et le décès un peu plus court que les autres: 21 mois vs 36 mois.

B) Astroblastomes infra-tentoriels: (à l'exclusion du tronc cérébral).

5 filles et 4 garçons sont inclus dans ce groupe et l'âge moyen est de 4 ans et 2 mois ( 11 mois à 11 ans et 8 mois). Le délai moyen jusqu'au diagnostic a été de 4 mois. 2 enfants n'ont pas été suivis dans notre centre et tous les autres sont décédés en moyenne 4,7 mois après l'établissement du diagnostic dont 3 en période post-opératoire.

\section{Le Médulloblastome}

Cette tumeur vient au second rang des néoplasmes de la fosse postérieure. Elle représente $13.7 \%$ de toute la série et approximativement le quart des tumeurs de la fosse postérieure. Nous en avons rencontré 27 cas dont 14 garçons et 13 filles. L'âge moyen était de 7 ans et 4 mois ( 1 an et 7 mois à 13 ans). Deux n'ont pas eu de follow-up. Le délai moyen jusqu'au diagnostic a été de 3 mois. La mortalité globale est importante $(72 \%)$ ainsi que la mortalité post-opératoire $(20 \%)$. Le délai moyen jusqu'au décès a été de 9,4 mois. Les 7 survivants $(28 \%)$ ont une évolution de 4 ans et 7 mois $(7$ mois à 8 ans).

Il faut noter que le traitement classique associant chirurgie et radiothérapie n'a pu être appliqué que chez 14 enfants. Un patient est décédé avant toute thérapie, 5 sont décédés en période post-opératoire et dans 5 autres cas, le traitement a été refusé. De ces 5 derniers patients, un seul survit 22 mois après l'établissement du diagnostic.

IV. Tumeurs du tronc cérébral.

Ce groupe comprend 26 cas. Il vient donc au troisième rang des tumeurs de la fosse postérieure.

Huit fois seulement nous avons eu un diagnostic histologique définitif: deu $x$ astrocytomes (D.M. 1 et 11 ), cinq astroblastomes (D.M. III et IV), et une tumeur sarcomateuse. Dans 18 cas, nous n'avons pu obtenir un diagnostic histologique.

Ce groupe est formé de 15 garçons et 11 filles dont l'âge moyen est de 7 ans et 4 mois (un an et 8 mois jusqu'à 20 ans). Le délai jusqu'au diagnostic a été de 4 mois ( 2 semaines à 12 mois). Chez 7 patients, nous n'avons pas de relance. Chez ceux dont l'évolution est connue, la mortalité est évidemment trés grande $(73.6 \%)$

8 cas ont bénificié de radiothérapie et ce traitement semble améliorer la survie. 4 enfants sont décédés mais les 4 autres sont encore vivants après un délai moyen de 2 ans et 8 mois. Deux toutefois présentent des signes de récidive. En ce qui concerne le groupe d'enfants non-traités, 10 sur $11(81 \%)$ sont décédés en moyenne 3,7 mois après l'établissement du diagnostic.

\section{Ependymomes.}

Ce diagnostic a été posé 6 fois chez 5 filles et 1 garçon dont l'âge moyen était de 3 ans et 7 mois ( 6 mois à 8 ans). Ces tumeurs étaient toutes situées au niveau de IVe ventricule. En dehors d'un enfant dont nous ne connaissons 
pas l'évolution, tous les patients sont décédés (dont un en période postopératoire), quel qu'ait été le traitement: chirurgie seule ou associée à de la radiothérapie. Le délai moyen jusqu'au décès a été de un an et 3 mois (une semaine à 2 ans et 5 mois).

\section{Le crânio-pharyngiome}

9 enfants $(4.6 \%$ de la série $)$ constituent ce groupe. Il y avait 6 filles et 3 garçons et l'âge moyen est de 10 ans $(6$ ans et 6 mois à 15 ans et 11 mois). Le délai entre l'apparition des premiers symptômes et le diagnostic a été en moyenne de 5 mois (de 1 à 14 mois).

6 des 9 enfants sont décédés $(66.6 \%)$ dont un en période post-opératoire. Les autres décès sont survenus en moyenne 11 mois après l'intervention chirurgicale ( 4 mois à 2 ans). 3 enfants survivent, respectivement 4 ans et 8 mois, 5 ans et 10 mois et 6 ans et 6 mois après le diagnostic et présentent tous trois des sequelles endocrinologiques contrôlables. Un a de plus une atrophie optique bilatérale avec diminution importante de l'acuité visuelle (20 sur 1000).

\section{DISCUSSION}

Au cours des 13 années que couvre cette étude, nous avons donc en moyenne traité 15 cas de tumeurs cérébrales par année. Il ne nous est pas possible à l'heure actuelle de tirer des conclusions définitives quant à l'incidence des lésions cérébrales tumorales dańs la population Canadienne-Française.

On estime généralement que l'incidence varie de 1.7 à 4 nouveaux cas par année par 100,000 enfants (Bergstrand et Coll., 1958; Gjerris, 1976; Schoenberg et Coll., 1975), ce qui, rappelons-le, classe ces tumeurs au premier rang des tumeurs solides de l'enfant et au deuxième rang des néoplasies après les leucémies (Young et Coll., 1975). La distribution selon les sexes est pratiquement égale dans notre série avec $51.8 \%$ de garçons et $48.2 \%$ de filles, ce qui diffère des données classiques où la prédominance des garçons est plus évidente (Gjerris, 1976: Matson, 1969). L'âge, en revanche, est superposable: $70 \%$ de la série est constituée par des enfants de la première décade.

La répartition selon la localisation avec $43.6 \%$ de lésions supratentorielles et $56.4 \%$ de lésions infratentorielles est également comparable à ce que lon retrouve dans certaines séries (Bergstrand et Coll., 1958; Gjerris, 1976; Marsden et Stewart, 1968) mais ne confirme pas la proportion habituellement rapportée en pédiâtrie de $2 / 3$ infra-tentorielles contre 1/ 3 supra-tentorielles (Matson, 1969; Bailey et Coll., 1939). Peut-être l'augmentation relative des tumeurs supra-tentorielles est-elle due à l'admission dans notre série de patients âgés jusqu'à 20 ans, puisqu'on sait que dans la deuxième décade le nombre de gliomes hémisphériques a tendance à augmenter (Rubinstein, 1972).

La fréquence des différents types histologiques rejoint généralement les chiffres cités dans la littérature médicale. Avec $80 \%$ environ de gliomes (astrocytomes, astroblastomes, épendymomes, médulloblastomes, gliomes optiques, oligodentrogliomes, pinéalomes) nous sommes un peu au-dessus des données de Bailey et Coll. (1939) mais très proches de celles de Smith et Coll. (1942), de Keith et Coll. (1949) et plus récemment, de Matson (1969) et Onoyama et Coll. (1975). Tout comme dans ces séries, l'astrocytome vient au premier rang aussi bien des lésions supra-tentorielles que de la fosse postérieure. On notera toutefois que les astroblastomes (degré de malignité III et IV) sont relativement nombreux $(13.7 \%$ du total) et égaux en nombre au médulloblastome qui est en fréquence, la deuxième tumeur de la fosse postérieure, après l'astrocytome et juste devant les tumeurs du tronc qui, dans notre série, sont au nombre de 26 ( $13 \%$ du total).

L'épendymome représente $3.7 \%$ des tumeurs que nous avons rencontrées, ce qui est moins fréquent que les chiffres rapportés par d'autres auteurs et où la fréquence varie de 9 à $12 \%$ (Gjerris et Coll., 1976; Matson, 1969, Onoyama et Coll., 1975; Rubinstein, 1972). Il en est de même pour le crâniopharyngiome $(5.5 \%)$ qui représente dans certaines séries jusqu'à $10 \%$ (22).
Les autres tumeurs non gliomateuses ont une fréquence sensiblement superposable à celles des études citées. Le petit nombre de ces tumeurs ne nous permet pas d'en donner, comme pour les néoplasies citées plus haut, un aperçu spécifique pour chacun des types.

En ce qui concerne la séméiologie, le tableau numéro 2 illustre les symptômes et signes cliniques notés au moment où le diagnostic a été posé. Il ne tient évidemment pas compte de l'ordre d'apparition des différents éléments cités. En revanche, nous avons pu établir pour chacun des groupes de tumeurs décrits, la durée moyenne de la symptomatologie avant l'établissement du diagnostic. On peut ainsi voir que les astrocytomes hémisphériques (D.M. I et II) ont une histoire habituellement plus longue que les astroblastomes soit de 6,5 vs 2,5 mois. De même, le crânio-pharyngiome a un délai de près de 6 mois. Par contre, les tumeurs infra-tentorielles présentent un délai généralement plus court entre 3 et 4 mois, quelque soit le type histologique impliqué. Ces constatations sont en accord avec les statistiques présentées par Gjerris (1976, 1978).

En excluant les néoplasies du tronc cérébral, les tumeurs de la fosse postérieure présentent la plus haute fréquence d'hypertension intracrânienne $(75 \%)$, nos chiffres étant toutefois légèrement inférieurs à ceux habituellement cités (Gjerris, 1976, 1978). Une obstruction rapide du IVe ventricule et les dimensions réduites de la fosse postérieure expliquent aisément cette symptomatologie. Pour la même raison, le syndrôme cérébelleux cinétique ou statique présent dans $60 \%$ des cas se comprend facilement. L'hypertension intracrânienne est en revanche beaucoup plus rare dans les tumeurs du tronc qui ont tendance à l'infiltration progressive avec refoulement de l'aqueduc de Sylvius et du IVe ventricule sans les bloquer. On comprendra dès lors qu'elles se manifestent plutôt par une atteinte des longs faisceaux et des nerfs crâniens, particulièrement le VIIe avec, à un degré moindre, un syndrôme cérébelleux.

L'hydrocéphalie et l'hypertension intra-crânienne sont moins fréquentes 
dans les tumeurs supra-tentorielles même si $20 \%$ d'entre elles sont situées sur la ligne médiane. Leur symptomatologie est plus diversifiée et en rapport avec la localisation et les régions lésées par la croissance tumorale.

Parmi les divers examens visant à dépister la présence d'une masse intracrânienne, il faut souligner la valeur de l'E.E.G. qui s'est avéré positif dans $78 \%$ des cas. Ce sont principalement les tumeurs du tronc cérébral qui échappent à cet examen. Les radiographies standards du crâne sont également un apport précieux, surtout pour confirmer l'hypertension intracrânienne (élargissement des sutures chez le nourrisson, déminéralisation de la selle turcique et empreintes digitiformes exagérées chez l'enfant plus âgé). Les calcifications ne sont par contre présentes que dans $7.5 \%$ des cas. Il est évident qu'actuellement, lả tomodensitométrie du cerveau assistée à l'ordinateur est devenue un élément très important de diagnostic, dont la fiabilité pourrait même devenir supérieure aux techniques conventionnelles de la neuroradiologie qui, dans notre étude, a donné des résultats positifs dans $81 \%$ des cas (Gawler et Coll., 1974, 1976; New et Coll., 1975).

Malgré qu'il soit difficile de présenter une analyse statistique rigoureuse de chaque groupe de tumeurs cérébrales, certaines grandes lignes propres à chacun des types histologiques peuvent être esquissées pour les tumeurs les plus fréquentes.

Ainsi, notre série confirme que l'astrocytome cérébelleux qui représente $17.2 \%$ des cas rencontrés (34 sur 197) est la tumeur cérébrale la plus fréquente en pédiatrie mais aussi la plus bénigne et celle dont le pronostic est le plus favorable puisque notre mortalité globale est de $13 \%$ et postopératoire de 3\% (Geissinger et Bucy, 1971; Gol et McKissock, 1959; Matson, 1956). Le pronostic de l'astrocytome supra-tentoriel est beaucoup plus dépendant de sa localisation, permettant ou non une exérèse chirurgicale radicale. De plus, les dimensions de ces tumeurs peuvent être énormes lorsqu'elle croissent dans des zones cérébrales neurologiquement muettes telles le lobe frontal. Elles peuvent évoluer longtemps (ici plus de 6 mois) entre les tout premiers symptômes et le moment du diagnostic. Par ailleurs, les diverses associations thérapeutiques (chirurgie et radiothérapie, radiothérapie et hormonothérapie) ne semblent pas influencer beaucoup sur la survie des malades (Pons et Coll., 1975). Un autre élément décisif dans le pronostic des gliomes est que nous pouvons confirmer le degré de malignité (Gjerris et Coll., 1976) tel que défini par Kernohan et Coll. (1949). Dans ce groupe où le degré de malignité IIl et IV a été vérifié histologiquement, la mortalité pour les 23 enfants dont l'évolution est connue a été de $100 \%$.

Le médulloblastome est une tumeur hautement maligne, survenant principalement au niveau du cervelet. Malgré l'association thérapeutique bien schématisée de la chirurgie et de la radiothérapie, le pronostic demeure réservé (Aron, 1971). Des guérisons sont cependant décrites, passée la période de risque de récidive assez clairement définie pour cette tumeur et qui serait égale à l'âge où le diagnostic a été posé, plus 9 mois, c'est-à-dire la durée de la grossesse (Quest et Coll., 1978). Nos taux de mortalité tant postopératoire que globale (respectivement 20 et $72 \%$ ) se rapprochent de ceux de la littérature (Aron, 1971; McFarland et Coll., 1969; Matson, 1969). La chimiothérapie récemment introduite dans le traitement de cette tumeur pourrait prolonger la survie, mais, à long terme, ne semble pas modifier le pronostic (Mealey et Hall, 1977).

La radiothérapie à haute dose $(5,000$ rads) et peut-être la chimiothérapie peuvent également prolonger la survie des patients présentant une tumeur du tronc cérébral (Lee, 1975; Villani et Coll., 1975). La chirurgie n'a, pour ce groupe de patients, qu'une place tout à fait secondaire se limitant à la dérivation en cas d'hydrocéphalie et à la ponction d'éventuels éléments kystiques. Même la biopsie ne semble plus requise lorsque le diagnostic neuro-radiologique est suffisamment clair. L'issue est toujours fatale et Matson suggère même de très fortement mettre en doute le diagnostic clinique de gliome du tronc si, après 18 mois, le malade est toujours en vie, qu'il y ait eu traitement ou non (Matson, 1969).

Le petit nombre d'épendymomes dans notre série ne nous permet pas de tirer de conclusions définitives. La localisation exclusive au niveau de IVe ventricule peut n'être qu'un hasard puisque l'on sait que cette tumeur se retrouve aussi bien en supra qu'en infra-tentoriel (Barone et Elvidge, 1970; Dohrmann et Coll., 1976). Notre taux de mortalité apparaît également plus élevé que celui de la littérature puisque Dohrmann et Coll. (1976) présentent une survie de $25 \%$ après 3 ans alors que Barone et Elvidge (1970), dans un groupe d'enfants et d'adultes, retrouvent $50 \%$ de survivants 5 ans après le diagnostic. Ces deux auteurs s'accordent à trouver un effet bénéfique à l'irradiation postopératoire.

Notre expérience dans le traitement du crânio-pharyngiome s'est avérée décevante avec une mortalité de $66 \%$ attribuable soit à une récidive de la tumeur, ou à des complications endocriniennes. Certains centres présentent des résultats thérapeutiques supérieurs, insistant sur la nécessité d'une excision aussi radicale que possible (Katz, 1975) et sur l'effet bénéfique de la radiothérapie postopératoire (Hoff et Patterson, 1972). Par contre, Svolos (1969), dans une étude extensive, présente des statistiques comparables aux nôtres quant au taux de mortalité.

\section{CONCLUSION}

La distribution des tumeurs cérébrales, tant du point de vue de leur localisation que de leur nature histologique, apparaît superposable, dans notre série, à celle décrite dans la littérature médicale. Pour certains types de tumeurs (astroblastomes, tumeurs du tronc, crânio-pharyngiomes, médulloblastomes), la mortalité demeure élevée malgré les progrés évidents de la neuro-chirurgie et de la radiothérapie. On peut espérer que, grâce à l'Emi-Scan, un diagnostic plus précoce pourra être possible. On peut aussi espérer que les progrès de la chimiothérapie et de l'immunothérapie, en ce qui concerne ces tumeurs, permettront d'en améliorer le pronostic (Ommaya, 1976; Walker et Weiss, 1975). 


\section{REMERCIEMENTS}

Nous désirons remercier les docteurs Michel Décarie et JeanPierre Mathieu du service de Neurochirurgie de l'hôpital SainteJustine pour leur collaboration et leur permission de consulter les dossiers de leurs patients. Nous voudrions aussi remercier les docteurs Raymond Lafontaine, Wanda Schiffman, Pierre C. David et Albert Larbrisseau du service de Neurologie de l'hôpital Sainte-Justine pour leurs conseils. Enfin, nous remercions aussi mesdemoiselles Lucille Tellier, Danielle Rousseau, Diane Tanguay et madame Marie Quessy pour l'aide secrétariale.

\section{REFERENCES}

AMBRAMSON, N.. RABEN, M.. CAVANAUGH, P. J. (1974). Brain tumours in children: Analysis of 136 cases. Radiology: 112: 669-672.

ARON, B. S. (1971). Medulloblastoma in children. Twenty-t wo year's experience with radiation therapy. Amer. J. Dis. Child. 121: 3|4-317.

BAILEY, P., BUCHANAN, D. N., BUCY, P. C. (1939). Intracranial tumours of infancy and childhood. Chicago: University of Chicago Press.

BARONE, B. M. ELFIDGE, A. R. (1970). Ependymomas. A clinical survey. $J$. Neurosurg. 33: 428-438.

BERGSTRAND, C. G., BERGSTEDT.J., HER RLIN, K. M. (1958). Paediatric aspects of brain tumours in infancy and childhood. Acta. Paediatrica Scandinav, 47: 688-698.

DOHRMANN, G. J.. FARWEL.I. J. R., FLANNERY, B. S. (1976). Ependymomas and ependymoblastomas in children. J. Neurosurg. 45: 273-283.

GAWLER, J., BULL, J. W., DU BOUIAY, G. H. (1974). Computer assisted tomography (EMI-Scanner). Its place in investigation of suspected intracranial tumours. Lancet: 2: $419-423$
GAWLER, J., DU BOULAY, G., BULL, J. W. B. (1976). A comparison of computer assisted tomography (EMI-Scanner) with conventionnal neuroradiologic methods in the investigation of patients suspected of intracranial tumour. J. Can. Assoc. Radiol. 27: $157-169$.

GEISSINGER, J. D., BUCY, P. C. (1971). Astrocytomas of the cerebellum in children. Long-Term study. Arch. Neurol. 24: 125135.

GJERRIS, F. KLEE, J. G. and KLINKEN, L. (1976). Malignancy grade and long-term survival in brain tumours of infancy and childhood. Acta Neurol. Scandinav. 53: 6171

GJERRIS. F. (1976). Clinical aspects and longterm prognosis of intracranial tumours in infancy and childhood. Develop. Med. Child Neurol. 18: 145-159.

GJERRIS. F. (1978). Clinical aspect and longterm prognosis of infratentorial intracranial tumours in infancy and childhood. Act. Neurol. Scandinav. 57: 31-52.

GOL, A.. MCKISSOCK. W. (1959). The cerebellar astrocytomas. A report on 98 verified cases. J. Neurosurg. 16: 287-296

HOFF. J. T. and PATTERSON. R. H. (1972). Craniopharyngiomas in children and adults. 3. Neurosurgery 36: 299-302.

KATZ. E. L. (1975). Late results of radical excision of craniopharyngiomas in children. J. Neurosurg. 42: 86-93.

KEITH, H. M., CRAIG, W. M., KERNOHAN J. W. (1949). Brain tumours in children. Pediatrics. 3: 839-844.

KERNOHAN, J. W., MABON, R. F., SVIEN H. J., ADSON, A. W. (1949). A simplified classification of gliomas. Proc. Staff Meetings Mayo Clinic. 24: 71-75.

LEE, F. (1975). Radiation of infratentorial and supratentorial brainstem tumours. J Neurosurg. 43: 65-68

MCFARLAND, D. R., HORWITZ, H.. SAENGER, EL (1969). Medulloblastoma A review of prognosis and survival. Br. J Radiol. 42: 198-214.

MARSDEN. H. B.. STEWART, J. K. (1968). Tumours in children. Berlin. Springer ed.

MATSON, D. D. (1956). Cerebellar astrocytoma in childhood. J. Pediatr. 18: 150-158.

MATSON, D. D. (1969). Neurosurgery of infancy and childhood 2nd Edition. p. 410479. Charles C. Thomas Publisher Springfield.
MEALEY, J. JR., HALL, P. V. (1977). Medulloblastoma in children. Survival and treatment. J. Neurosurg. 46: 56-64.

NEW. P. J. F.. SCOTT, W. R. SCHNUR. J. A (1975). Computed tomography with the EMI-Scanner in the diagnosis of primary and metastatic neoplasms. Radiology 114: 75-87.

OMMAYA, A. K. (1976). Immunotherapy of gliomas: A review. In Advances in Neurology. Vol. 15 pp. 337-349, Raven Press.

ONOYAMA, Y.. ABE, M., TAKAHASHI, M., YABUMOTO, E.. SAKAMOTO, T., SAKAMOTO, T. (1975). Radiation therapy of brain tumors in children. Radiology 115 : 687-693.

PONS, A, ARMAND, J P. COMBES, P. F. NADAL. P. (1975). Survie des malades atteints de tumeurs cérébrales sustentorielles primitives en fonction des associations thérapeutiques. Neurochirurgie: 21: 408-410.

QUEST, D. O., BRISMAN, R., ANTUNES, J. L., HOUSEPIAN, E. M. (1978). Period of risk for recurrence in medulloblastoma. J. Neurosurg. 48: 159-163.

RUBINSTEIN, L. J. (1972). Tumors of the central nervous system. In Atlas of Tumor Pathology, fasc. 6 Published by the Armed Forces Institute of Pathology.

SCHOENBERG, B. S., SCHOENBERG,D. C., CHRISTINE, B. W., GOMEZ, R. R (1975). The epidemiology of primary intracranial neoplasms of childhood: A population study. Neurology. 25: 256.

SMITH, W. A, FINCHER, E. F. (1942). Intracranial tumors in children: Preliminary study of 100 cases. South Med. J. 35: 547554

SVOLOS. D. G. (1969). Craniopharyngiomas: A study based on 108 verified cases.

VIL.LANTI, R., GAINI, S. M., TOMEI, G. (1975). Follow-up study of brain-stem tumors in children. Child's Brain I: 126-135.

WALKER, M. D. WEISS, H. D. (1975) Chemotherapy in the treatment of malignant brain tumors. In Advances in Neurology. Vol. 13, pp. 149-191. W. J. Friedlander edit. Raven Press.

YOUNG, J-L. Jr., MILLER, R. W. (1975) Incidence of malignant tumors in U.S children. J. Pediat, 86: 254-258. 